\title{
Numerical study on the thermohydraulic performance of a reciprocating room temperature active magnetic regenerator
}

\author{
Georges El Achkar ${ }^{1, *}$, Bin Liu ${ }^{1}$, and Rachid Bennacer ${ }^{1,2}$ \\ ${ }^{1}$ Tianjin Key Laboratory of Refrigeration Technology, Tianjin University of Commerce, Guangrong Rd 409, Beichen District, Tianjin, \\ 300134, China \\ ${ }^{2}$ LMT/ENS-Cachan/CNRS, Paris-Saclay University, 61 Avenue du Président Wilson, 94235 Cachan, France
}

\begin{abstract}
In this paper, the thermohydraulic performance of a reciprocating room temperature active magnetic regenerator (AMR), with gadolinium (Gd) particles used as a magnetocaloric material (MCM) and water used as a working fluid, was numerically investigated. A two-dimensional transient flow model was developed using COMSOL Multiphysics, in order to determine the water flow distribution in two AMRs of cross and parallel Gd particles distributions for different water inlet velocities of $0.06 \mathrm{~m} . \mathrm{s}^{-1}, 0.08 \mathrm{~m} . \mathrm{s}^{-1}, 0.1 \mathrm{~m} . \mathrm{s}^{-1}$ and $0.12 \mathrm{~m} . \mathrm{s}^{-1}$. The Gd particles have a radius of $1.5 \mathrm{~mm}$ and a distance from one another of $0.9 \mathrm{~mm}$. Based on the simulations results of the first model, a two-dimensional transient coupled flow and heat transfer model was then developed using COMSOL Multiphysics, in order to characterise the convective heat transfer in the AMR of cross Gd particles distribution for the same water inlet velocities.
\end{abstract}

\section{Introduction}

The growing need for cooling, refrigeration and air conditioning requires an increasing use of two-phase thermal systems with mechanical vapour compression, such as heat pumps, capillary pumped loops or loop heat pipes. Nevertheless, these thermal systems have the disadvantage of operating with refrigerants, which often have a negative impact on the ozone layer and/or a significant contribution to the greenhouse effect. The quality of the new environmentally friendly refrigerants will probably be to the detriment of the energy performance or the stability of these thermal systems. It is thus important to search for new cold production solutions ensuring low environmental impact and high energy performance and stability.

In this context, the magnetic refrigeration technology, based on the magnetocaloric effect (MCE) of MCMs, appears as a potentially viable solution [1-3]. Since the theoretical optimal coefficient of performance (COP) of magnetic refrigeration systems is of the same order as that of two-phase thermal systems and their operation occurs with solid materials coupled with water, the possibilities seem to fit with the current requirements. In addition, this new technology operates at low pressure and frequency, which is positive for the security, the maintenance cost, the noise and the lifetime.

The MCE is a physical phenomenon that occurs in a MCM under the influence of a varying magnetic field $(H)$ [4-7]. It is usually expressed as an adiabatic temperature change $\left(\Delta T_{a d}\right)$ or an isothermal total entropy change $\left(\Delta S_{t o t}\right)$ of a MCM. In the absence of a magnetic field $(H=0)$, the magnetic moments in the MCM are disordered. If

*e-mail: georges.elachkar@tjcu.edu.cn a magnetic field is applied to the MCM $(H>0)$, the magnetic moments are forced to align in a higher order. As a consequence, the magnetic entropy $\left(S_{m}\right)$ decreases. In isentropic (i.e. adiabatic) conditions, the total entropy $\left(S_{t o t}=S_{m}+S_{l}\right)$ remains constant. Therefore, the decrease of the magnetic entropy manifests itself in an increase of the lattice entropy $\left(S_{l}\right)$. The atoms in the MCM start to vibrate more intensively, and as a consequence, its temperature increases. The opposite occurs when the magnetic field is removed $(H=0)$. The MCM magnetic entropy increases and its temperature decreases. This effect is maximal around the Curie temperature which marks the change between the ferromagnetic state and the paramagnetic state of the MCM. This transition is the most frequently studied so far.

It is well known that the MCE of most MCMs at moderate magnetic fields (up to $1.5 \mathrm{~T}$ ) is limited to a maximum $\Delta T_{a d}$ of $5 \mathrm{~K}[8,9]$. This value is not sufficient for such MCMs to be directly implemented into a practical cooling device, where a much greater temperature span could be required. Therefore, in order to increase the temperature span, the best solution is to integrate an AMR in the magnetic refrigeration system. The operation cycle of this AMR is generally based on four thermodynamic processes (Figure 1):

- Adiabatic magnetisation (phase 1): The MCM initially at room temperature is subjected to a magnetic field, causing its uniform temperature increase according to its adiabatic temperature change $\Delta T_{a d}$;

- Isomagnetic entropy transfer (phase 2): A cool working fluid circulation is imposed through the MCM, always in presence of the magnetic field. The working fluid 
recovers the heat from the MCM and hence the working fluid heats up and the MCM cools down;

- Adiabatic demagnetisation (phase 3): The working fluid circulation is stopped and the magnetic field is removed, causing an uniform decrease of the MCM temperature (below the room temperature).

- Isomagnetic entropy transfer (phase 4): A cool working fluid circulation is imposed through the MCM, always in absence of the magnetic field. The working fluid releases the heat to the MCM and hence the working fluid cools down and the MCM heats up.

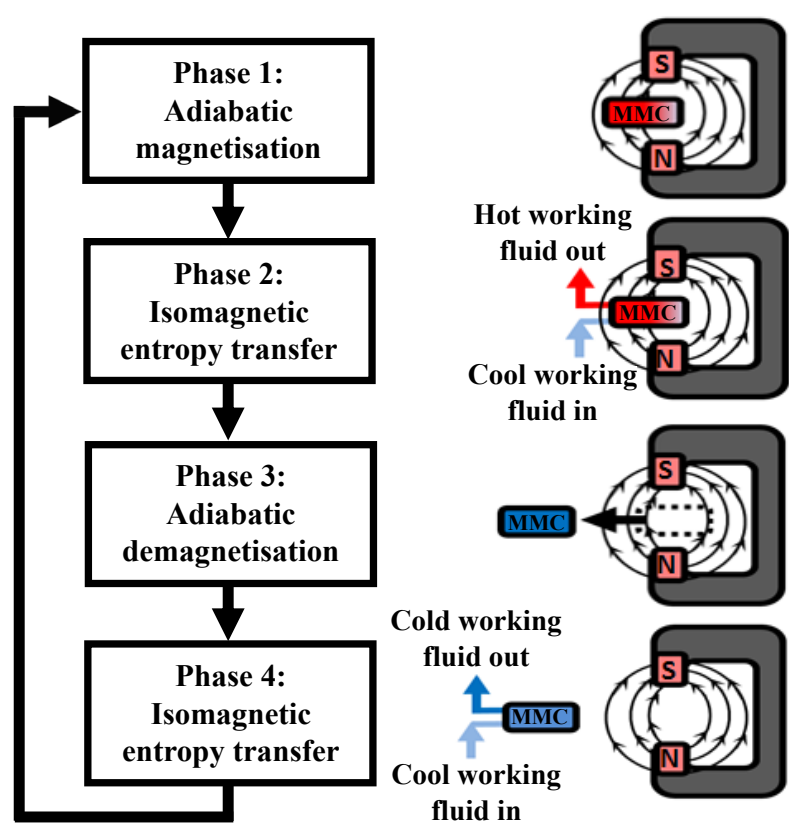

Figure 1. Schematic diagram of the AMR operation cycle.

In this work, the thermohydraulic performance of a reciprocating room temperature AMR was numerically investigated by determining the water flow distribution and the convective heat transfer for different operating conditions.

\section{Numerical simulations}

\subsection{Physical model}

The physical model of the magnetocaloric system, consisted of an AMR and a movable permanent magnet, is presented in figure 2 .

The AMR was splitted into two symmetrical cylinders (Figure 3), in order to enhance its heat transfer efficiency. These cylinders were designed according to the flow between the MCM particles configuration (Figure 4) presented by Kawanami et al. [10]. The permanent magnet alternatively moves towards the left and right cylinders, inducing their alternative magnetisation and demagnetisation. When the permanent magnet covers one cylinder, the magnetised MCM of this cylinder heats up, whereas the demagnetised MCM of the other cylinder cools down.
Likewise, the water alternatively flows in the two directions through the AMR during the phases 2 and 4 to exchange heat with the MCM.

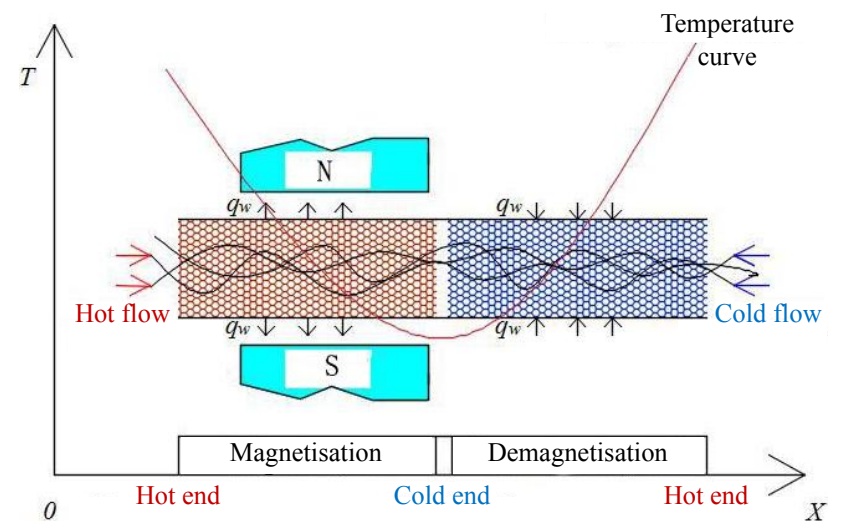

Figure 2. Physical model of the magnetocaloric system.

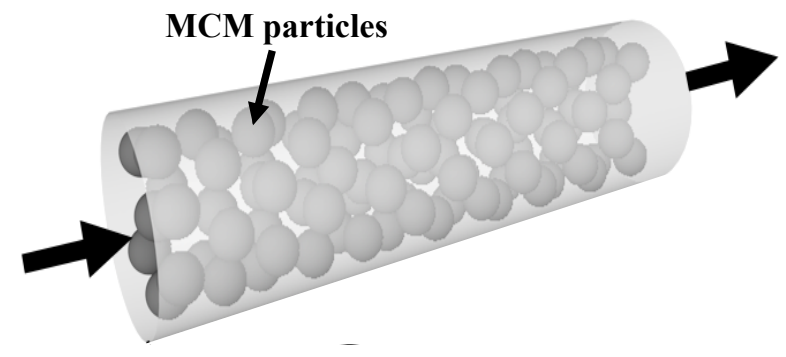

Figure 3. Schematic diagram of the AMR.

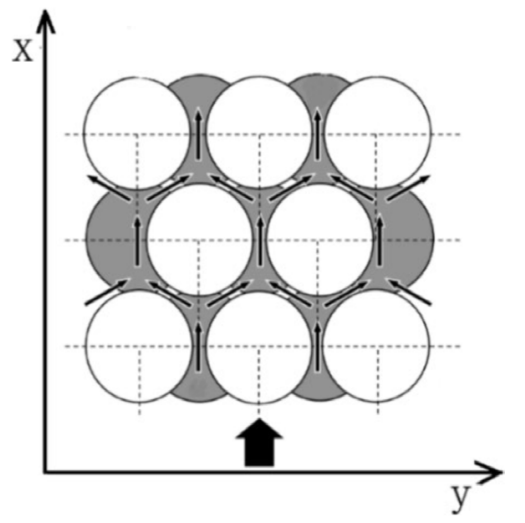

Figure 4. Flow model configuration of Kawanami et al. [10].

\subsection{Mathematical flow model}

Based on this physical model, a two-dimensional transient flow model was developed using COMSOL Multiphysics [11], in order to determine the water flow distribution in the AMR.

\subsubsection{Assumptions}

In order to simplify the model, the following assumptions were considered: 
- The working fluid is incompressible;

- The working fluid flow is laminar;

- No slip condition at the particle surface;

- The thermophysical properties of the MCM and the working fluid remain constant regardless of their temperature and the magnetic field;

- The singular pressure drop of the working fluid at the AMR inlet is neglected.

According to these assumptions, the mathematical model can be written as follows:

- Momentum equations in $\mathrm{x}$-axis and $\mathrm{y}$-axis directions:

$$
\begin{gathered}
\rho_{w f}\left(u \frac{\partial u}{\partial x}+v \frac{\partial u}{\partial y}\right)=-\frac{\partial p_{w f}}{\partial x}+\mu_{w f}\left(\frac{\partial^{2} u}{\partial x^{2}}+v \frac{\partial^{2} u}{\partial y^{2}}\right) \\
\rho_{w f}\left(u \frac{\partial v}{\partial x}+v \frac{\partial v}{\partial y}\right)=-\frac{\partial p_{w f}}{\partial x}+\mu_{w f}\left(\frac{\partial^{2} v}{\partial x^{2}}+v \frac{\partial^{2} v}{\partial y^{2}}\right)-\rho_{w f} g
\end{gathered}
$$

- Continuity equation:

$$
\frac{\partial u}{\partial x}+\frac{\partial v}{\partial y}=0
$$

For the calculation of the AMR hydraulic diameter $D_{h}$, the following correlation corresponding to a similar particles structure proposed by Vuarnoz and Kawanami [12] was used:

$$
D_{h}=\frac{2(2 \sqrt{3}-\pi)}{\pi} r_{0}
$$

Knowing the hydraulic diameter, the inlet water velocity in the AMR can be calculated by:

$$
U_{w f}=\frac{m_{w f}}{\rho_{w f} S}
$$

\subsubsection{Simulations conditions}

The conditions considered in the simulations are reported in table 1. Two parallel and cross MCM particles distributions were considered in the AMR. The water mass flow rate was chosen so that the Reynolds number $R e_{w f}$ is lower than 2000, in order to ensure a laminar flow.

Table 1. Simulations conditions - Part 1

\begin{tabular}{cc}
\hline Parameter (unit) & Value \\
\hline $\mathrm{S}\left(\mathrm{m}^{2}\right)$ & 0.0008 \\
$\mu_{w f}\left(\mathrm{~kg} \cdot \mathrm{m}^{-1} \cdot \mathrm{s}^{-1}\right)$ & $1.006 \times 10^{-3}$ \\
$\lambda_{M C M}\left(\mathrm{~W} \cdot \mathrm{m}^{-1} \cdot \mathrm{K}^{-1}\right)$ & 10.6 \\
$c_{p M C M}\left(\mathrm{~J}^{-1} \mathrm{~kg}^{-1} \cdot \mathrm{K}^{-1}\right)$ & 235.485 \\
$\mathrm{r}_{0}(\mathrm{~mm})$ & 1.5 \\
$\mathrm{~T}_{0}(\mathrm{~K})$ & 291.15 \\
$\mathrm{p}_{\text {out }}(\mathrm{Pa})$ & 0 \\
$\mathrm{U}_{w f}\left(\mathrm{~m} \cdot \mathrm{s}^{-1}\right)$ & $0.06 ; 0.08 ; 0.1 ; 0.12$ \\
\hline
\end{tabular}

\subsubsection{Results and discussion}

Inlet Velocity: $0.06 \mathrm{~m} / \mathrm{s} \quad$ Time: $0.7 \mathrm{~s}$

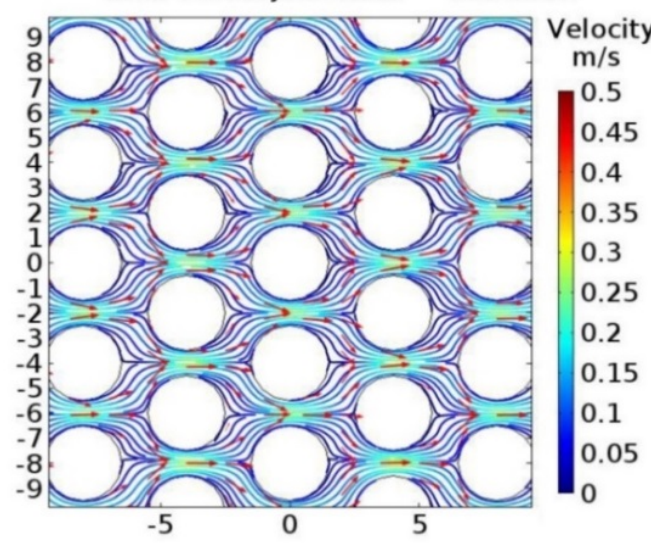

Inlet Velocity: $0.08 \mathrm{~m} / \mathrm{s} \quad$ Time: $0.7 \mathrm{~s}$

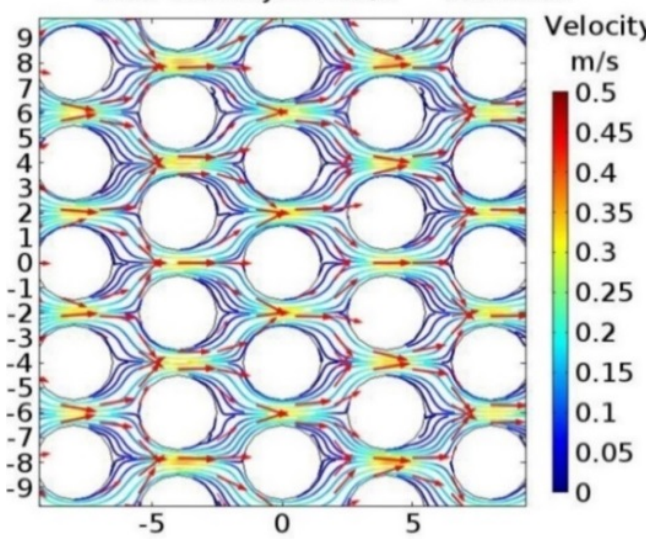

Inlet Velocity: $0.1 \mathrm{~m} / \mathrm{s} \quad$ Time: $0.7 \mathrm{~s}$

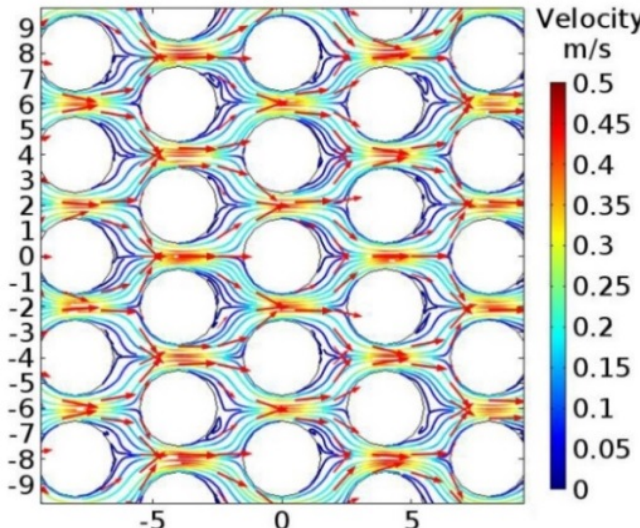

Inlet Velocity: $0.12 \mathrm{~m} / \mathrm{s}$ Time: $0.7 \mathrm{~s}$

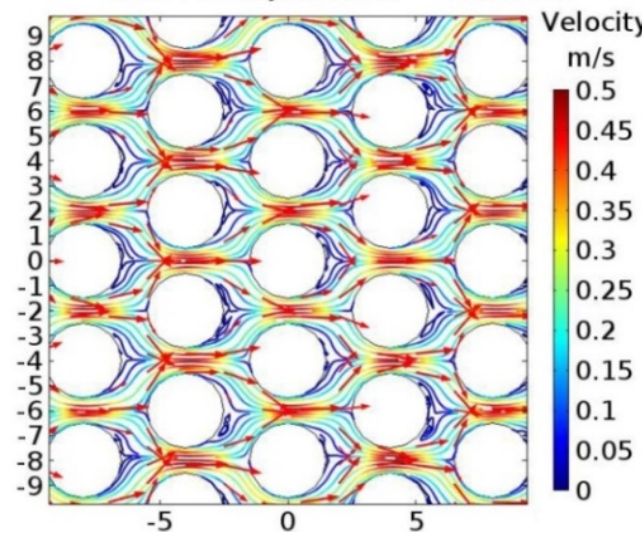

Figure 5. Water flow distribution in the AMR of cross MCM particles distribution for different water inlet velocities. 


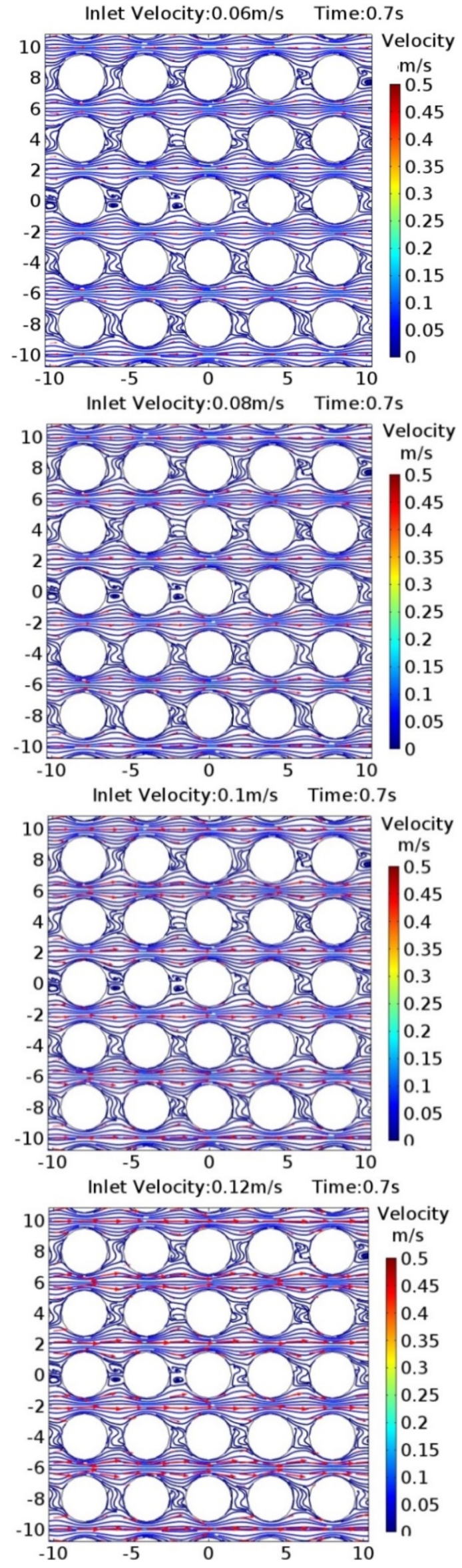

Figure 6. Water flow distribution in the AMR of parallel MCM particles distribution for different water inlet velocities.
Figure 5 shows the water flow distribution in the AMR of cross MCM particles distribution for different water inlet velocities. It can be found that, for water inlet velocities of $0.06 \mathrm{~m} . \mathrm{s}^{-1}$ and $0.08 \mathrm{~m} . \mathrm{s}^{-1}$, no Eddy flow was generated in the AMR. For water inlet velocity higher than 0.1 $\mathrm{m} . \mathrm{s}^{-1}$, the Eddy flow obviously appeared in the right half of the AMR. This phenomenon caused the water to stay in the convective heat transfer zones, which is not conducive to the heat transfer process. Indeed, when the water flows around the spherical surface of the particle, the flow is accelerated in the left half of the curved particle surface and the streamlines outside the boundary layer can be considered as a potential flow. The flow velocity between the particles is relatively high and the pressure is low. The water pressure in the boundary layer is consistent with the flow direction, and its gradient overcomes the viscous force between the water and the curved surface. The water in this interval is not separated from the boundary layer, and hence, it can fully exchange heat with the particle. When the fluid flows through the right half of the curved particle, the water velocity decreases and the pressure increases in the flow direction. Under the action of the back pressure gradient, the water reflows and pushes its particles away from the curved surface, which leads to a formation of a boundary layer separation. Therefore, for a two-dimensional flow model with MCM particles of radius $1.5 \mathrm{~mm}$ and of distance $0.9 \mathrm{~mm}$ from one another, the optimal water inlet velocity was about $0.1 \mathrm{~m} . \mathrm{s}^{-1}$ for a convenient convective heat transfer between the particles and the water.

Similarly, figure 6 shows the water distribution in the AMR of parallel MCM particles distribution for different water inlet velocities. It can be found that the main water flow passes in the $y$-axis direction through the space between the particles, and a small water flow passes in the $\mathrm{x}$-axis direction through the gap between the particles. Therefore, the heat exchange between the particles and the water mainly occurs through the upper and lower surfaces of the particles. Compared with the cross MCM particles distribution structure, this structure cannot effectively transfer the heat from the particles to the water, reducing thus the heat transfer performance of the AMR.

Figure 7 shows the evolution of the water pressure gradient in the $\mathrm{x}$-axis direction as a function of the water inlet velocity in the AMRs of cross and parallel MCM particles distributions. The overall trends of the pressure gradient curves in the two cases are similar, however, the pressure gradient in the cross MCM particles distribution case is significantly higher than that of the parallel MCM particles distribution case. This can be explained by the greater complexity of the cross MCM particles distribution configuration, inducing higher singular and frictional pressure drop. 


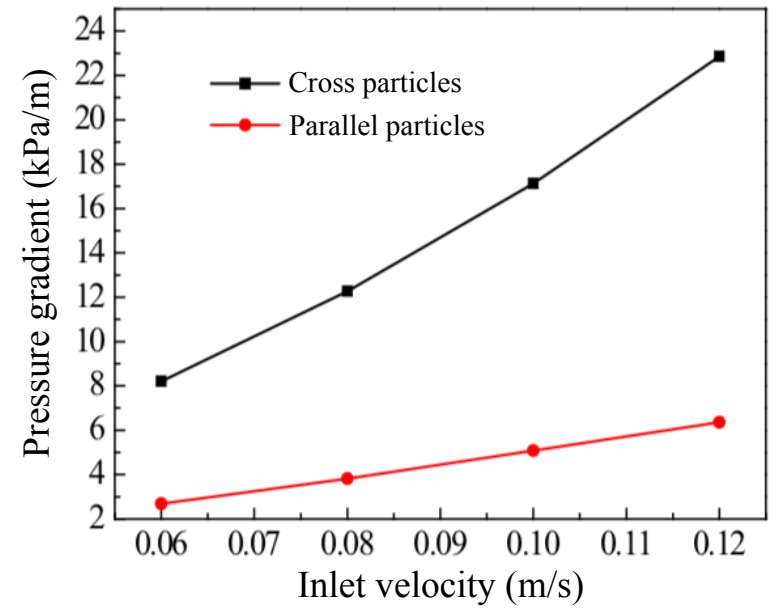

Figure 7. Evolutions of the water pressure gradient in the $\mathrm{x}$-axis direction as a function of the water inlet velocity in the AMRs of cross and parallel MCM particles distributions.

\subsection{Mathematical flow and heat transfer model}

Based on the simulations performed on the water flow distribution, a two-dimensional transient coupled flow and heat transfer model was then developed using COMSOL Multiphysics, in order to characterise the convective heat transfer in the AMR. The governing equation is expressed by:

$$
\frac{\partial(\rho \phi)}{\partial t}+\frac{\partial(\rho u \phi)}{\partial t}+\frac{\partial(\rho v \phi)}{\partial t}=\frac{\partial}{\partial x}\left(\Gamma \frac{\partial \phi}{\partial x}\right)+\frac{\partial}{\partial y}\left(\Gamma \frac{\partial \phi}{\partial y}\right)+S T
$$

\subsubsection{Assumptions}

In order to simplify this model, the following assumptions were considered:

- The working fluid is incompressible;

- No slip condition at the particle surface;

- The thermophysical properties of the MCM and the working fluid remain constant regardless of their temperature and the magnetic field;

- The thermal processes in the AMR occur under adiabatic boundary conditions;

- The heat losses are neglected.

\subsubsection{Simulations conditions}

For the $\Delta T_{a d}$ of the $\mathrm{Gd}$, the experimental measurement method was adopted. When the $\mathrm{Gd}$ is at a room temperature of $18^{\circ} \mathrm{C}$ under a magnetic field of $1.5 \mathrm{~T}$, the measured $\Delta T_{a d}$ was equal to $3.5^{\circ} \mathrm{C}$. On the basis of the flow model, the heat source inside the MCM particles was added. The inlet water temperature was $18^{\circ} \mathrm{C}$ and the Prandtl number was 7.5. For the AMR of cross MCM particles distribution, different inlet velocities of $0.06 \mathrm{~m} . \mathrm{s}^{-1}, 0.08 \mathrm{~m} . \mathrm{s}^{-1}$, $0.1 \mathrm{~m} . \mathrm{s}^{-1}$ and $0.12 \mathrm{~m} . \mathrm{s}^{-1}$ were chosen to simulate the convective heat transfer process.

\subsubsection{Results and discussion}

Figure 8 shows the temperature distribution in the AMR of cross MCM particles distribution for water inlet velocity of $0.1 \mathrm{~m} . \mathrm{s}^{-1}$ and temperature of $18{ }^{\circ} \mathrm{C}$ at initial time (before the water flow in the heated MCM) and time of 0.5 s.

(a) $t=0 s$

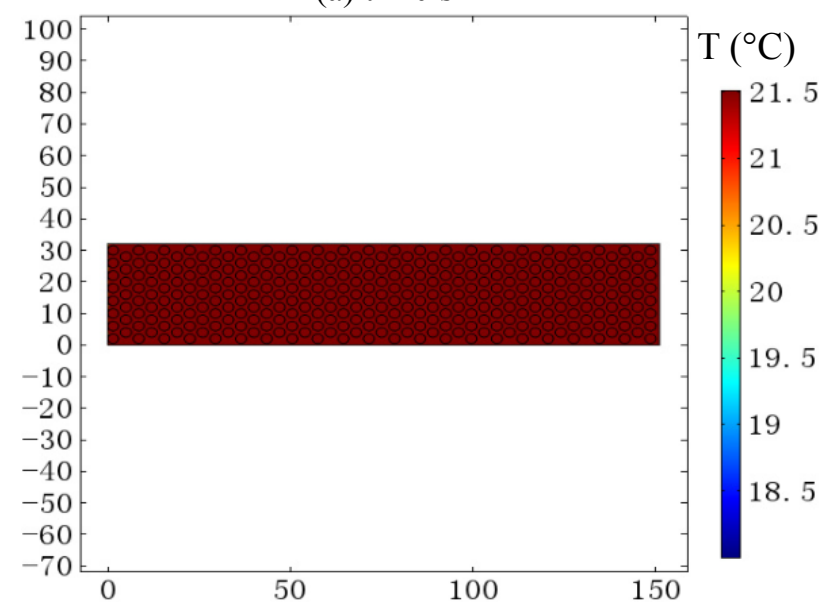

(b) $\mathrm{t}=0.5 \mathrm{~s}$

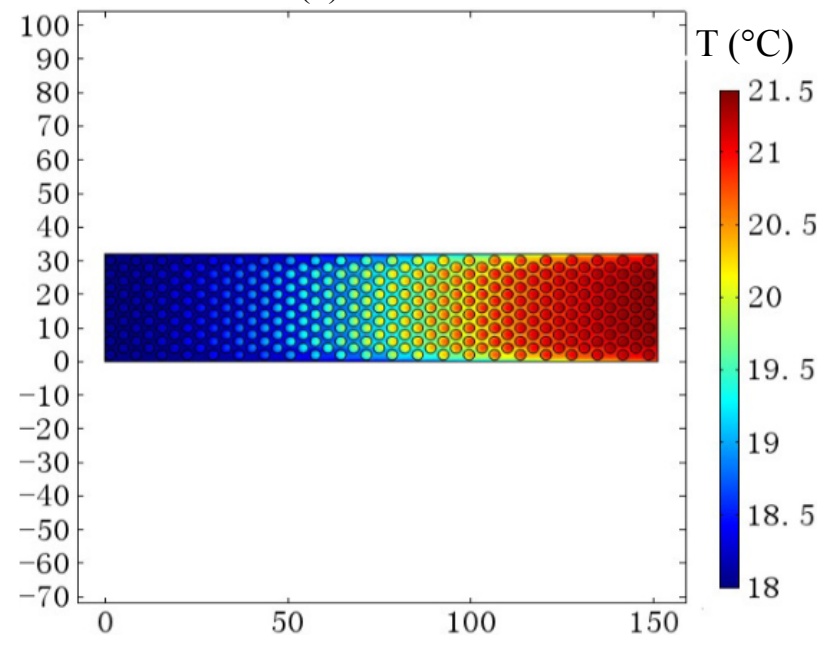

Figure 8. Temperature distribution in the AMR of cross MCM particles distribution for water inlet velocity of $0.1 \mathrm{~m} . \mathrm{s}^{-1}$ and temperature of $18{ }^{\circ} \mathrm{C}$ at (a) $t=0 \mathrm{~s}$ and (b) $t=0.5 \mathrm{~s}$.

It was found that the temperature distribution at the AMR inlet was uniform. When the water flowed around the particles along the AMR, its temperature close to the particles raised and the temperature of the particles back surface was relatively high because of the water back-flow. The break up of the thermal boundary layer caused by the Eddy phenomenon enhanced the heat transfer between the MCM and the water. However, the energy was trapped due to the relatively short convective heat transfer time, as shown in figure 9 , and the convective heat transfer was weakened. Besides, the increase of the water inlet velocity leaded to an increase of the convective heat transfer.

Figure 10 shows the evolutions of water velocity as a function of the position in the $\mathrm{x}$-axis direction around the MCM particle for different inlet velocities. The water velocity 
trend was qualitativement the same for all the inlet velocities. The flow first accelerated in the particle left half and then decelerated in the right particle half, as found previously. Owing to the boundary layer separation caused by the presence of the Eddy flow, the water velocity in the $\mathrm{x}$-axis direction approached to zero.

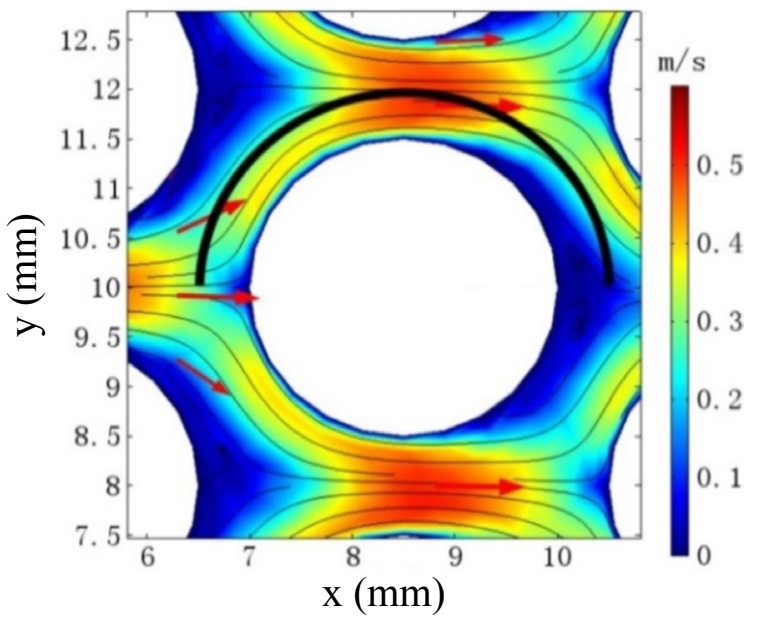

Figure 9. Water velocity distribution around a MCM particle.

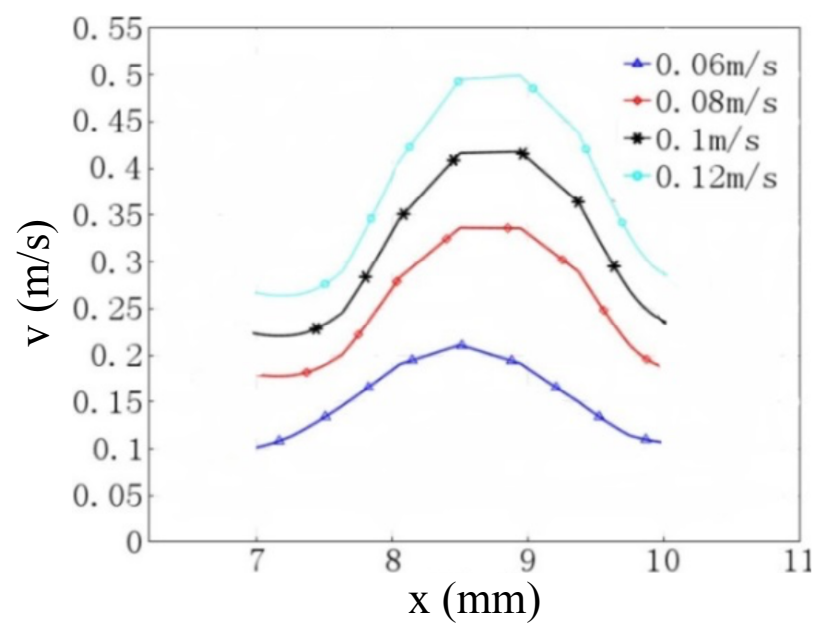

Figure 10. Evolutions of water velocity as a function of the position in the $\mathrm{x}$-axis direction around a MCM particle for different water inlet velocities.

In order to further analyse the convective heat transfer process around the particles, the Nusselt number $\mathrm{Nu}$ in the $\mathrm{x}$ axis direction was analysed and calculated at the same position of the water velocity shown previously (Figure 11). First, the Prandtl was calculated by the one-dimensional plot group in the COMSOL Multiphysics software. The $\mathrm{Nu}$ was calculated by the water flow velocity curve and the Prandtl curve near the surface of the particle as follows:

$$
N u=0.35\left(\frac{S_{1}}{S_{2}}\right)^{0.2} \operatorname{Re}_{w f}^{0.6} \operatorname{Pr}_{w f}^{0.36}\left(\frac{P r_{w f}}{P r_{w}}\right)^{0.25}
$$

Where $S_{1}=4 \mathrm{~mm}$ and $S_{2}=3.5 \mathrm{~mm}$.

Figure 11 shows the evolutions of the Nusselt Number as a function of the position in the $\mathrm{x}$-axis direction around the MCM particle for different water inlet velocities. It can be seen that the $N u$ first increased in the particle left half and then decreased in the right particle half regardless of the water inlet velocity, which is coherent with the trend of the water velocity. Besides, an increase of the water inlet velocity increased the local velocity and hence the local $\mathrm{Nu}$. The best way to enhance the convective heat transfer between the particles and the water is thus, other than increasing the heat exchange surface, to increase the inlet velocity and the mixing of the water.

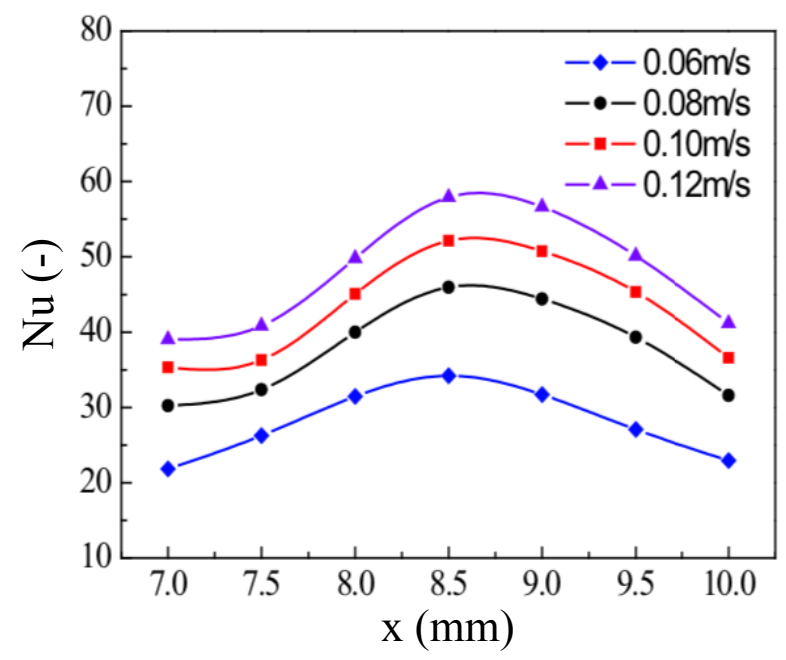

Figure 11. Evolutions of Nusselt Number as a function of the position in the $\mathrm{x}$-axis direction around a MCM particle for different water inlet velocities.

\section{Conclusions and perspectives}

A numerical study on the thermohydraulic performance of a reciprocating room temperature AMR was conducted. Two-dimensional transient flow and coupled flow and heat transfer models were developed using COMSOL Multiphysics, in order to characterise the flow and the convective heat transfer in two AMRs of parallel and cross MCM particles distributions. The water flow distribution and pressure drop in the AMR were determined in the two configurations showing that the cross MCM particles distribution was more advantageous for the water mixing and the break up of the thermal boundary layer, which leads to an enhancement of the convective heat transfer.

As perpectives to this work, different AMR geometries and MCMs will be studied in order to optimise the convective heat transfer while ensuring a lowest pressure drop possible.

\section{Acknowledgments}

Financial support from the National Natural Science Foundation of China (No. 51706154) is gratefully acknowledged. 


\section{Nomenclature}

$D_{h}$

g

$m$

$N u$

$p$

$\operatorname{Pr}$

$r_{0}$

Re

S

$S T$

$t$

$T$

$u$

U

v

$c_{p}$

$\Gamma$

$\lambda$

$\mu$

$\phi$

$\rho$

\section{Subscripts}

MCM

out

$w$

$w f$

0 hydraulic diameter of the AMR, $m$ gravity acceleration, $\mathrm{m} \cdot \mathrm{s}^{-2}$

mass flow rate at AMR inlet, $\mathrm{kg} . \mathrm{s}^{-1}$

Nusselt number, -

relative pressure, $\mathrm{Pa}$

Prandtl number, -

particles radius, $m$

Reynolds number, -

cross-sectional area of the AMR, $\mathrm{m}^{2}$

source term, $\mathrm{J}$

time, $s$

initial temperature, ${ }^{\circ} \mathrm{C}$

velocity in $\mathrm{x}$ direction, $\mathrm{m} . \mathrm{s}^{-1}$

inlet velocity, $\mathrm{m} . \mathrm{s}^{-1}$

velocity in y direction, $\mathrm{m} . \mathrm{s}^{-1}$

heat capacity, $\mathrm{J}_{\mathrm{kg}}{ }^{-1} \cdot \mathrm{K}^{-1}$

thermal diffusion coefficient, $\mathrm{m}^{2} \cdot \mathrm{s}^{-1}$

thermal conductivity, $\mathrm{Wm}^{-1} \cdot \mathrm{K}^{-1}$

dynamic viscosity, $\mathrm{kg} \cdot \mathrm{m}^{-1} \cdot \mathrm{s}^{-1}$

general variable, -

density, kg.m ${ }^{-3}$

magnetocaloric material

outlet

wall

working fluid

initial

\section{References}

[1] S.L. Russek, C.B. Zimm, International Journal of Refrigeration 28, 1366 (2006)

[2] O. Sari, M. Balli, International Journal of Refrigeration 37, 8 (2014)

[3] N.A. Mezaal, K.V. Osintsev, T.B. Zhirgalova, IOP Conference Series: Earth and Environmental Science 87, 032024 (2017)

[4] E.G. Warburg, Annalen der Physik (Leipzig) 13, 141 (1881)

[5] P. Weiss, A. Piccard, Journal de Physique Théorique et Appliquée 7, 103 (1917)

[6] A. Smith, The European Physical Journal H 38, 507 (2013)

[7] M.W. Zemansky, Dover Publications (1981)

[8] K.A. Gschneidner Jr, V.K. Pecharsky, A.O. Tsokol, Reports on Progress in Physics 68, 1479 (2005)

[9] E. Brück, O. Tegus, D.T.C. Thanh, K.H.J. Buschow, Journal of Magnetism and Magnetic Materials 310, 2793 (2007)

[10] T. Kawanami, K. Chiba, K. Sakurai, M. Ikegawa, International Journal of Refrigeration 29, 1294 (2006)

[11] S. Whitaker, Transport in Porous Media 1, 3 (1986)

[12] D. Vuarnoz, T. Kawanami, Applied Thermal Engineering 37, 388 (2012) 